\title{
AN OO BASED METHODOLOGY FOR REAL-TIME HW/SW SYSTEMS MODELING
}

\section{O.P. Dias ${ }^{1}$, I.M. Teixeira², J.P. Teixeira², C.E. Pereira $^{3}$}

The purpose of this paper is to present a methodology for modeling, at system level, complex real-time hw/sw systems. The methodology makes use of an object-oriented modeling technique. A method is proposed to integrate the time dimension at system level specification. The method adapts some concepts of UML. The quality of the architectural solution is assessed in terms of timing constraints, performance, testability and diagnosability. The main features of the methodology are ascertained through an example.

\section{Introduction}

Specification and design of hardware/software systems, supported on heterogeneous technologies within the hardware (hw) and software (sw) domains, pose difficult challenges $[1,2]$. If the time dimension is considered, at system level, those difficulties further increase. The need of formal methodologies for hw/sw system description at architectural level, as well as the adequacy of Object-Oriented (OO) paradigm [3] and of CASE-like tools for accomplishing such needs is pointed out in [4]. It is also pointed out as desirable that "architecture performance evaluators will evolve to general evaluators of system performance". The authors in [5] and [6] have also pointed out those aspects, and proposed solutions for the raised problems.

A significant $R \& D$ effort is being undertaken in the last decade, both from academia and vendors, in the area of $\mathrm{hw} / \mathrm{sw}$ systems specification, design, and simulation. The inclusion of the time variable, at system level specification of hw/sw systems, is not a trivial task [7,8]. In fact, most of the available literature on real-time systems specification refers, mainly, to sw systems [9-13]. For instance, some useful

\footnotetext{
${ }^{1}$ Escola Superior de Tecnologia/Instituto Politécnico de Setúbal, INESC, Portugal

${ }^{2}$ Instituto Superior Técnico (IST), Universidade Técnica de Lisboa, INESC, Portugal

${ }^{3}$ Universidade Federal do Rio Grande do Sul, Porto Alegre, Brasil
} 
guidelines for such inclusion are provided in $[12,13]$ making use of data flow analysis techniques [14] and of object-oriented models [13].

The purpose of this paper is to introduce a methodology for modeling, at system level, complex real-time hw/sw systems. The paper is organized a follows. In section 2 , the methodology is presented. Section 3 describes the proposed technique to introduce the time variable, at system level. Section 4 details the metrics used for the assessment of the quality of the architectural solution. Section 5 describes a case study, which demonstrates the usefulness of the proposed methodology. Finally, section 6 summarizes the conclusions.

\section{Methodology}

Referring to $\mathrm{OO}$ paradigm, which is the basic modeling technique in our methodology, guidelines provided in [13] assume that objects and corresponding scenarios of behavior are already identified. However, UML points out use-case diagrams, identifying the main system functionalities and the corresponding responsible actors, as crucial tools to the design and the development processes. In this work, we follow this approach, and start the design process with use-case diagrams. This approach allows system architects to start at higher levels of abstraction, searching for a near-optimum object definition. That is, instead of starting, directly, with the static view of an $\mathrm{OO}$ model, we decompose high-level usecase diagrams in lower-level use-case diagrams, which allow us to identify functionality that is not immediately assigned to the already identified objects. This functional description of the problem under analysis corresponds, in fact, to its DFD ${ }^{1}$ (Data Flow Diagram) representation [15]. In this context, a DFD corresponds to a usecase functional description. This is the approach proposed in this work. Thus, the DFD representation of use-case diagrams is a basic construct of the proposed methodology for architectural design and quality assessment. In fact, as it will be shown in section 3, it allows us to introduce functionality and time at system level specification. In fig 1 (a) and (b) an abstract example is depicted to highlight this approach.

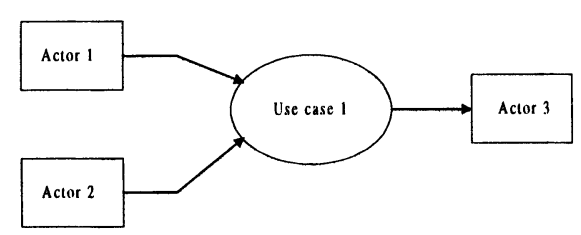

(a)

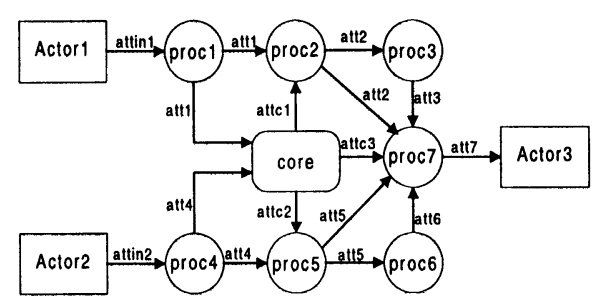

(b)

Fig 1 (a) Abstract use-case diagram; (b) Processes within use-case

The proposed methodology aims at: (1) the inclusion of the time dimension at systemlevel specification, in order to encompass real-time hw/sw systems; (2) the generation of hw/sw system architectures that meet pre-defined functional, performance and test requirements $[8,18]$. As referred, the first step of the proposed methodology is applied 
to the DFD description of a given problem. Based on this DFD, a first directed graph (PAG, Process and Attributes Graph) is derived, in which vertices are attributes and/or processes (Fig. 2 (a), corresponding to example in fig.1). In PAG, edges represent either attribute update through a process, or a necessary input of an attribute to a process. In order to use powerful graph partitioning tools, like Chaco [16], a second graph (TOG, Task-Oriented Graph) is built. In TOG, vertices are tasks. A task is defined as the association of a process and the attribute updated by it. Hence, atomic processes are assumed [15]. Chaco analyses the graph (e.g., Fig. 2 (b)), in order to achieve a task clustering that satisfies a minimal-cut criterion. Such criterion guarantees that the resulting objects exhibit moderate cohesion, coupling and autonomy ${ }^{3}$, which are, as will be shown, key features, both from the design and test points of view. According to a pre-specified number of graph partitions, clustering result is mapped into objects. Chaco is embedded in SysObj [6], a CASHE (Computer Aided Software Hardware Engineering) tool under development. As a result, for each number of pre-specified objects, an $\mathrm{OO}$ model, which is, in fact, a possible solution for system architecture, is obtained. Criteria and quality metrics have been formalized (section 4), and are, then, used, through SysObj, for assessing architecture quality.

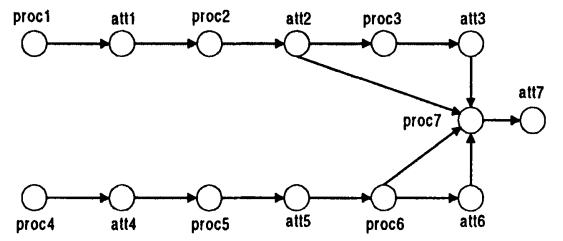

(a)

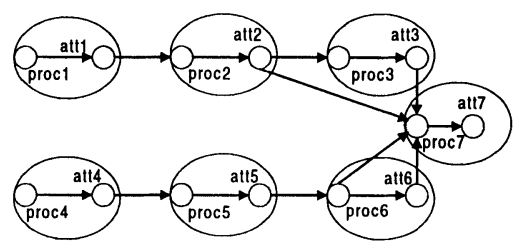

(b)

Fig. 2 (a) PAG Graph; (b) TOG Graph

\section{Inclusion of Real Time Constraints}

In order to include the time dimension, at system level specification, we assume that task complexity [8] may be expressed in terms of the time required to its execution. At this stage, complexity is formally quantified by assigning weights to the tasks. The partitioning algorithm of Chaco allows task weighting, which can be user's defined. Moreover, we propose that the unit of time may correspond to delay units, to clock units or to real-time units. By doing so, it is possible to model different types of systems.

Task execution ends when the corresponding attribute is up-dated. Two different mechanisms are introduced to assign weights to tasks. In the first mechanism, a weight is attributed to the process within a task, reflecting the intrinsic complexity of the process. The second mechanism consists of introducing time constraints to the attributes [8], thus simulating sequences, or interrupts of task execution. To achieve this purpose, we sequence the attribute update, either by associating the attribute to a number that represents its position in the sequence rank, or by describing the attribute in connection with a condition (or a guard, in OO technology). 
At this phase of the hw/sw design process, weights can only be predicted, as implementation (either in hw and/or in sw) is yet to be defined. If it is not possible to reach an architectural solution that complies with real-time requirements, then the design task is restarted. The initial DFD is, hence, modified, by assigning the required functionality to other task distribution. Atomic processes are, as referred, assumed, which eases process testability and diagnosability.

An extension of the methodology proposed in $[18,19]$ is used to derive architectural solutions. Now, the methodology carries out task clustering according to the weights assigned to the tasks. Task clustering is carried out in order to obtain a balanced (of equivalent complexity) aggregation of tasks. Aggregated tasks are mapped into objects leading to an architectural solution. The association to each task of the time required to its execution, thus constrains the resulting architecture. Attribute and task sequencing determines the time schedule for object activation, thus modeling realtime systems process. As the min-cut algorithm of Chaco is used, concurrent features are obtained, namely moderate cohesion, coupling and autonomy, for system objects. Consequently, balanced dynamic performance is achieved, thus allowing the exploitation of parallelism both in normal operation and during test sessions.

Different architecture solutions, satisfying real-time constraints, can also be obtained, by fixing the maximum complexity allowed for task clustering into objects. Additional, customer requirements may introduce constraints to object autonomy. This is the case, e.g., of using real-world objects such as low-cost microprocessors for hw implementation. Moreover, it may be necessary to modify this maximum complexity value until pre-defined quality criteria, in terms of functionality, performance, testability and diagnosability are satisfied by the resulting architecture.

\section{Quality Metrics}

For evaluating the quality of each solution three quality metrics are proposed, namely, object autonomy, object cohesion and coupling among objects [19]. Object autonomy, defined as the ratio between the number of edges inside an object, and the number of PAG edges reaching or leaving an object is an indicator of object dependence on the remaining objects. Object cohesion, defined as the ratio between the actual number of PAG edges within an object and the maximum number of edges that the object could contain without changing its attributes and methods, is an indicator of object complexity. Coupling among objects is defined as the ratio between the number of objects to which the object under analysis sends data or control, over the total number of remaining objects in the system. Coupling between objects reflects the strength of association established by a connection from one entity to another. By using it, we can estimate the influence of an object over the remaining objects within the system.

In order to decide about the quality of a given solution, based on the values obtained for autonomy, cohesion and coupling, criteria are required. In fact, as it is shown through the case study (section 5), there are no standard values that correspond to a "good" architecture. These values are bounded between two limits, (1) no clustering, leading to an elementary architecture (EA) having as many objects as tasks; and (2) 
full clustering, leading to a complex architecture (CA) having a single object containing all the tasks. For intermediate clustering, quality metrics are evaluated with SysObj, using the formal definition of the different metrics, as proposed in $[18,19]$. As an example of metric formulation, for an architecture with $n$ objects, and discriminating between control and data attributes, control autonomy metric, $C A t_{x_{-} k}$, for object $\mathrm{x}$ and index $\mathrm{k}$, and average control autonomy metric, ACAt, are defined as:

$$
\begin{gathered}
C A t_{x_{-} k}=\frac{\sum_{i=1}^{n_{O A x_{x}-k}} \sum_{j=1}^{n_{O M_{x_{-}} k}} d_{o_{T}}\left(a_{i, j}\right)+\sum_{i=1}^{n_{O A_{x_{-}-k}}} \sum_{j=1}^{n_{O M_{X_{-}-k}}} d_{i_{T}}\left(a_{i, j}\right)+2 \times \sum_{i=1}^{n_{O A_{x_{-}} k}} \sum_{j=1}^{n_{O M_{x_{-}} k}} d_{T}\left(a_{i, j}\right)}{c c e_{x_{-} k}} \\
A C A t=\frac{\sum_{k=1}^{n_{S O}} C A t_{x_{-} k}}{n_{S O}}
\end{gathered}
$$

where $n_{O A_{x_{-} k}}\left(n_{O M_{x_{-} k}}\right)$ is the number of attributes (methods) of object $x_{-} k ; a_{i, j}$ is the generic element of the Process and Attribute Matrix $\left(\mathrm{PAM}^{2}\right)$ describing the relationship among methods, $j$, and attributes $i ; c c e_{x_{-} k}$ represents the number of object $\mathrm{x} \_\mathrm{k}$ control edges that establish connection with other objects; $d_{o_{C}}\left(a_{i, j}\right)\left\{d_{i_{C}}\left(a_{i, j}\right)\right\}$ represent the $(-1)\{(+1)\}$ elements of the PAM. These edges diverge \{converge $\}$ from vertice $j$ (method) and converge \{diverge\} to vertice $i$ (attribute). If $a_{i, j}=-1 \quad\left\{a_{i, j}=+1\right\}$, then $d_{o_{C}}\left(a_{i, j}\right)=1 \quad\left\{d_{i_{C}}\left(a_{i, j}\right)=1\right\}$; $d_{C}\left(a_{i, j}\right)$ represent the (1) elements of the PAM. These edges identify eventual feedback in the graph. If $a_{i, j}=1$, then $d_{C}\left(a_{i, j}\right)=1$.

Using the metrics, formal criteria are required to guide the decision on what is the architectural solution that best fits system requirements. We propose the following criteria:

$$
\begin{aligned}
& \left.n_{S O} \ll n_{S P} \text { (especially if } n_{S P} \gg 1\right) \\
& n_{S O}{ }^{\ni} A C l\left(n_{S O}\right) \leq 1 \\
& n_{S O}{ }^{\ni} \operatorname{ACoh}\left(n_{S O}\right)=\overline{A \operatorname{Coh}\left(n_{S O}\right)} \\
& n_{L} \min
\end{aligned}
$$

Here, $\mathrm{ACl}$ and $\mathrm{ACoh}$ are, respectively, the average autonomy and cohesion associated with each architecture, $n_{S O}$ and $n_{S P}$ are the number of system objects and system processes, respectively, and $n_{L}$ is the number of loops among objects in the resulting architecture. The reasons for proposing these criteria are as follows: Condition (4), quantifying a experimentally verified heuristic, corresponds to the acceptance of objects with, at most, equal number of edges inside them than reaching/leaving them. 
Condition (5) guarantees, in principle, that i) object complexity is not too high, not to low and ii) if it is necessary to include new functionality, this can be done without unmanageable increase of complexity. Condition (6) guarantees low and easily controllable coupling among objects. In fact, object loops should be avoided, to avoid time conflicts and to increase testability. Condition (1) gives a first estimate on $\mathrm{n}_{\text {SO }}$. Experimental results show that these criteria usually lead to the high-quality architectures.

Another relevant feature of the quality of a given architecture is its testability. Hence, a set of testability oriented metrics and criteria are proposed. These metrics assess object controllability and observability, which are characteristics that quantifies the ability to reach object inputs and outputs. A formal definition of some of these metrics is $[18,19]$

Object Controllability

$$
O C t r_{x_{-} k}=\frac{\text { piafp }}{i a f p}
$$

Object Observability

$$
\text { oObs }_{x_{-} k}=\frac{\text { poafp }}{\text { oapf }}
$$

Average System Controllability

Average System Observability

$$
A S C t r=\frac{\sum_{k=1}^{n_{S O}} O C t r_{x_{-} k}}{n_{S O}}
$$

$$
A S O b s=\frac{\sum_{k=1}^{n_{S O}} O O b s_{x_{-} k}}{n_{S O}}
$$

where, piafp is the number of primary input attributes; iafp is the number of input attributes; poafp is the number of primary output attribute and oapf is the number of output attributes; $n_{S O}$ is the number of system objects. In the case study described below it is shown how these metrics can be used in order to guide the choice of the most adequate architecture.

\section{Case Study}

In order to ascertain the features of the proposed methodology, a generic system constituted by 8 processes and 8 actors is considered. The corresponding DFD is depicted in fig 3. Based on this DFD, different architectures can be obtained with the proposed methodology, as a result of different partitions carried out by SysObj and Chaco. In order to decide which is the architecture that best fits a given set of constraints, metrics and criteria are used as decision parameters, according to the procedure described in the previous section. 


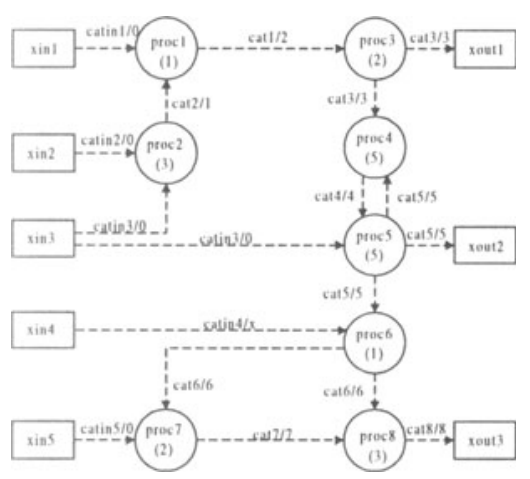

Fig 3 Data flow diagram of the generic system under study.

\subsection{Decision parameters}

For the DFD of Fig. 3, four different architectures are generated, corresponding to different $n_{S O}$. As referred, main decision parameters are average cohesion, system coupling, average autonomy and number of loops among objects (see Fig. 4).

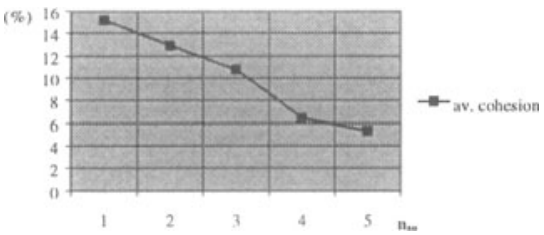

(a)

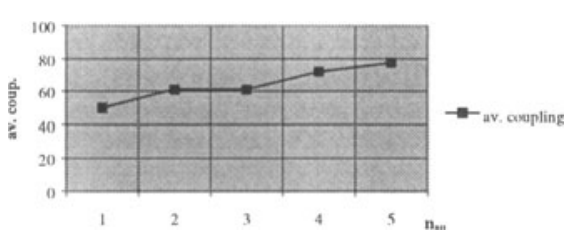

(b)

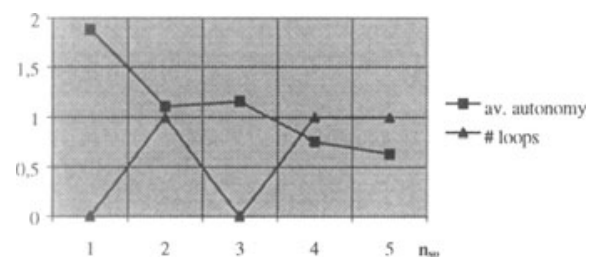

(c)

Fig. 4 (a) Average cohesion (b) System coupling

(c) Average autonomy and number of loops among objects

As it can be observed, the 3-object architecture presents several advantages over the others solutions. In fact, it presents moderate coupling and cohesion, which is indicated by the equidistance among each of these values and the extreme values of the corresponding curve. Moreover it satisfies criterion (4) for the autonomy metric, and presents no loops among objects. In Fig. 5, process clustering for 3-object architecture solution is shown. The correspondent instance diagram is depicted in Fig 6. 


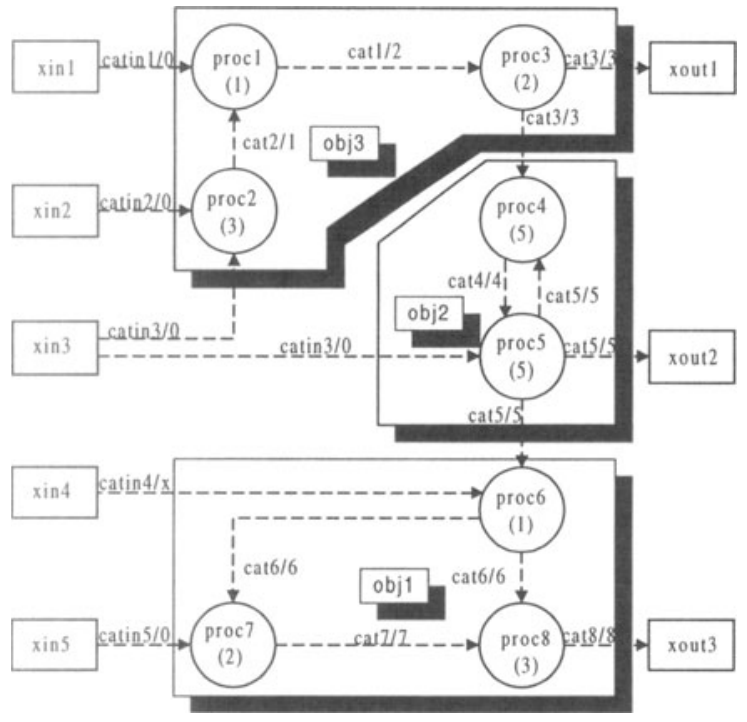

Fig. 5 Selected architecture;

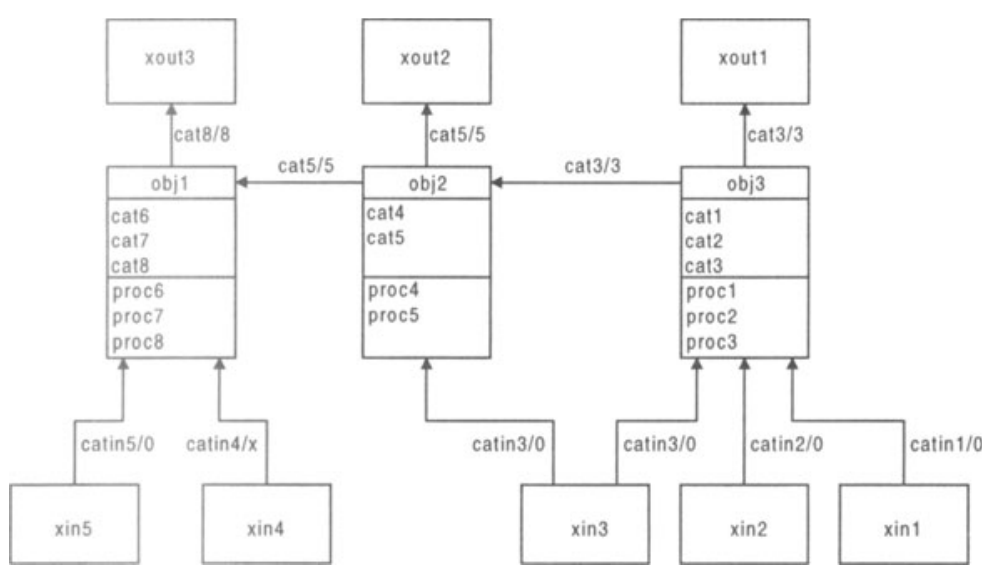

Fig. 6 Instance Diagram 


\subsection{Testability issues}

As referred, testability issues should be taken into consideration in the choice of the best fitted architecture. In table 1, values for the different metrics are represented for the architecture solutions generated by SysObj. From a performance point of view, the key metrics for choosing a "good" architecture are autonomy and number of loops, whose values (Fig. 4) are repeated in this table to higlitght their correlation with testability oriented metrics, controlability and observability.

As can be observed in Table 1, the 3-object architecture solution may be chosen also as a result of using testability criteria. In fact, it does not correspond to maximum average values of controllability and observability; however, it presents the best tradeoff between average and standard deviation (SD) values, and autonomy and number of loops. As a matter of fact, slightly better values for object controllability and observability are obtained with the 2-object architecture solution. However, it presents 1 loop among objects, while the 3-object solution presents no loops. Moreover, the 2object solution may lead to objects which may be too complex to test.

\begin{tabular}{|c|c|c|c|c|c|c|c|}
\hline nso & av. control. & SD contr. & av. observ. & SD obser. & av. auton. & SD auton. & \# loops \\
\hline \hline 2 & $37.5 \%$ & $12.5 \%$ & $41.6 \%$ & $8.3 \%$ & 1.1 & 0.1 & 1 \\
\hline 3 & $41.6 \%$ & $14.3 \%$ & $38.8 \%$ & $7.9 \%$ & 1.2 & 0.2 & 0 \\
\hline 4 & $37.5 \%$ & $22.7 \%$ & $41.6 \%$ & $36.3 \%$ & 0.8 & 0.5 & 1 \\
\hline 5 & $33 \%$ & $29.3 \%$ & $46.6 \%$ & $45.2 \%$ & 0.6 & 0.5 & 1 \\
\hline
\end{tabular}

Table 1- Object Controllability and Observability: average values and standard deviations (SD).

\section{Conclusions}

In conclusion, a methodology for the generation of real-time hw/sw system architectures has been proposed. The time dimension is introduced in the DFD corresponding to a use-case functional description, through task weighting. As a consequence, the time variable constrains the final architecture solution. The SysObj tool automatically generates a limited subset of possible architectures, and quality metrics and criteria are introduced for architecture quality evaluation. Both design and test features of the proposed architectures are taken into account. The design team is, hence, supported in the decision process, leading to a target 'best-fitted' architecture. A case study has been presented, that shows the usefulness of the proposed methodology.

\section{Endnotes}

'It is well known that a DFD doesn't convey information about process hierarchy or time sequence. In fact, it is, merely, a planar (at a given level of detail) representation of data and data transformation through processes. 
${ }^{2}$ PAM is the description of the directed graph (representing the DFD), in which vertices are processes and/or attributes and edges represent either attribute update through a process, or a necessary input of an attribute to a process.

${ }^{3}$ These concepts are defined on section 4 .

\section{References}

[1] D.D.Gajski et. al. "Specification and Design of Embedded Systems", Prentice Hall, 1994.

[2] L. Lavagno, A. Sangiovanni-Vincentelli, H. Hsieh, "Embedded System Codesign", Nato ASI-Series, Kluwer Academic Publishers, 1996.

[3] J.Rumbaugh, "OMT papers", 94/95, http://www.rational.com/support/techpapers/omt/.

[4] "System Design Technology Roadmap", Europe's Road to the Future, Version Final, Jan., 1998.

[5] M. Calha, J.P. Teixeira, I.C. Teixeira, "HW/SW Specification Using OOM Techniques", Proc. of the 7th IEEE Int. Workshop on Rapid Systems Prototyping, pp. 96-101, 1996.

[6] O.P. Dias, I.C. Teixeira, J.P. Teixeira, "Architectural Quality Evaluation for High-Level DFT", Proc. Int. IFIP Workshop on Logic and Architectural Synthesis (IWLAS), pp. 185 193, Grenoble, December, 1997.

[7] B. Selic, B, G. Gullekson, P. Ward, " Real-Time Object-Oriented Modeling", John Wiley \& Sons, Inc., ISBN 0-471-59917-4, 1994.

[8] M. Awad, J. Kuusela, J. Ziegler, "Object-Oriented Technology for Real-Time Systems: A Practical Approach using OMT and Fusion", Prentice Hall, 1996.

[9] J. Gwinn, "Object-Oriented Programs in Real-time", ACM SIGPLAN Notices, Vol. 27, No. 2, Feb 1992, 1992.

[10] R.S. Pressman, "Software Engineering. A Practitioner's Approach" Fourth Edition, MacGrawHill, 1997.

[11] J. Rumbaugh, M. Blaha, W. Premerlani, F. Eddy and W. Lorensen, "Object-Oriented Modeling and Design", Prentice Hall, 1991.

[12] “UML - Unified Modeling Language”, version 1.0, Rational Software Corporation, January, 1997, .http://www.rational.com.

[13] G. Booch, "Object-Oriented Design with Applications", The Benjamin/Cummings Publishing Company, Inc., 1994.

[14] C. Pereira, A. Frigeri, P. Darscht, W. Halang. "Object-Oriented Development of RealTime Industrial Automation Systems", IFAC Triennial World Congress, San Francisco USA, pp. 321-326. ISBN:0-08042923-8, July 1996.

[15] O. P. Dias, I. C. Teixeira J. P. Teixeira, C.E. Pereira,'Design and Quality Assessment of Real-Time Hw/Sw Systems Architectures", accepted for presentation at the Design of Integrated Circuits and Systems Conference (DCIS), Madrid, Nov. 1998.

[16] B. Hendrickson, R. Leland, "The Chaco User's Guide - Version 2.0", Technical Report SAND95-2344, Sandia National Laboratories, Albuquerque, NM 87185-1110, July 1995.

[17] "Complexity Measurements", http://www.mccabe.com/appnote/complex.html.

[18] O. P. Dias, I. C. Teixeira J. P. Teixeira, C.E. Pereira,"Performance and Test Oriented Metrics and Criteria for Hw/Sw Design at System Level", Proceedings of Design and Diagnostics of Electronic Circuits and Systems Workshop (DDECS), pp. 219-225, 1998.

[19] O. P. Dias, I. C. Teixeira, J. P. Teixeira,'Metrics and Criteria for Quality Assessment of Testable Hw/Sw Systems Arquitectures", accepted for inclusion in the special issue of the Journal of Electronic Testing : Theory and Applications (JETTA), to appear at the end of 1998. 